\title{
The set-shifting profiles of anorexia nervosa and autism spectrum disorder using the Wisconsin Card Sorting Test: a systematic review and meta-analysis
}

\author{
H. Westwood ${ }^{1}$, D. Stahl ${ }^{2}$, W. Mandy ${ }^{3}$ and K. Tchanturia ${ }^{1,4 *}$ \\ ${ }^{1}$ Psychological Medicine, King's College London, Institute of Psychiatry, Psychology \& Neuroscience, London, UK \\ ${ }^{2}$ Department of Biostatistics, King's College London, Institute of Psychiatry, Psychology \& Neuroscience, London, UK \\ ${ }^{3}$ University College London, Research Department of Clinical, Educational and Health Psychology, London, UK \\ ${ }^{4}$ Ilia State University, Tbilisi, Georgia
}

\begin{abstract}
Difficulties in set-shifting are commonly reported in both autism spectrum disorder (ASD) and anorexia nervosa (AN) populations. Despite this, it is not known whether this cognitive profile persists across different ages, or whether the profiles seen in ASD and AN are comparable. This systematic review and meta-analyses aimed to compare the set-shifting profiles, as measured by the Wisconsin Card Sorting Test (WCST) in adults and younger people with either ASD or AN, relative to healthy controls (HCs) and to statistically compare performance on the WCST between ASD and AN. In all, 24 studies on ASD and 22 studies on AN were identified. In ASD, there were significant differences between the clinical group and HCs, with the ASD group making significantly more perseverative errors, indicating greater difficulty in set-shifting [pooled effect size of $d=0.67,95 \%$ confidence interval (CI) $0.53-0.81, p \leqslant 0.001$ ]. This effect was consistent across the age span. For AN studies, there was a significant difference between adults with AN and HCs $(d=0.52$, $95 \%$ CI $0.36-0.68, p \leqslant 0.001)$ but a non-significant effect in child studies $(d=0.25,95 \%$ CI -0.05 to $0.55, z=1.66, p=0.096)$. Meta-regression indicated no effect of diagnosis (AN or ASD) on performance in adult studies but there was a nonsignificant trend $(p=0.053)$ towards children with ASD performing worse than children with AN. While difficulties with set-shifting appear to be stable in ASD, there may be differences between children and adults with AN, which warrant further investigation.
\end{abstract}

Received 7 July 2015; Revised 9 February 2016; Accepted 16 February 2016; First published online 25 April 2016

Key words: Anorexia nervosa, autism spectrum disorder, meta-analyses, set-shifting, Wisconsin Card Sorting Test.

\section{Introduction}

A growing body of literature indicates a link between anorexia nervosa (AN) and autism spectrum disorder (ASD). A systematic review of the prevalence of ASD in AN reported a mean rate of $23 \%$ (Huke et al. 2013); however, six of the included studies came from the same Swedish community sample and this line of research is ongoing (Nielsen et al. 2015). There are also similarities in cognitive style between AN and ASD (Gillberg et al. 2010; Oldershaw et al. 2011; Courty et al. 2013; Tchanturia et al. 2013a) reported in the literature. Although elevated levels of autistic traits have consistently been found within AN populations (Baron-Cohen et al. 2013; Tchanturia et al. 2013b; Huke et al. 2014; Rhind et al. 2014; Westwood et al. 2016) it is not known whether the

* Address for correspondence: K. Tchanturia, Psychological Medicine, King's College London, Institute of Psychiatry, Psychology \& Neuroscience, London, UK and Ilia State University, Tbilisi, Georgia.

(Email: kate.tchanturia@kcl.ac.uk) presence of these traits represents a shared, underlying mechanism (Zucker et al. 2007; Odent, 2010; Allely, 2013) or whether individuals with AN come to resemble those with ASD due to the chronic, starved state associated with the disorder (Pellicano \& Hiller, 2013; Treasure, 2013). Direct comparison of the seemingly shared traits, as well as systematic exploration of these traits across stages of illness, e.g. childhood and adulthood, will be essential to understanding the extent to which these traits may be shared mechanisms in both AN and ASD.

Interest in the potential relationship between AN and ASD has led to examination of cognitive traits, believed to underpin ASD in AN. While some traits associated with ASD, such as emotional theory of mind, have been found to reduce in recovered AN groups (Oldershaw et al. 2010), other traits including difficulties with set-shifting, or flexible thinking, appear to persist after weight gain (Tchanturia et al. 2004; Gillberg et al. 2010; Danner et al. 2012; Lindner et al. 2014). Recent reports suggest that set-shifting inefficiencies are present in children with $\mathrm{AN}$ who have had a relatively short duration of illness (Lang

This is an Open Access article, distributed under the terms of the Creative Commons Attribution licence (http://creativecommons.org/licenses/by/4.0/), which permits unrestricted re-use, distribution, and reproduction in any medium, provided the original work is properly cited. 
et al. 2015a) and relatives with AN (Holliday et al. 2005; Roberts et al. 2010; Lang et al. 2015b). Conversely, fasting in healthy controls (HCs) has been found to reduce set-shifting test performance, suggesting that starvation may exacerbate underlying difficulties in this domain (Bolton et al. 2014; Pender et al. 2014). Thus, despite evidence of cognitive similarity between AN and ASD, the extent and nature of this likeness remain unclear. Exploring differences in the set-shifting profiles of children and adults with AN would speak to debates of whether neurocognitive traits such as setshifting underlie the eating disorder or whether these cognitive traits are more related to eating disorder pathology such as starvation.

Differences in set-shifting have been observed between children and adults with AN. A systematic review of set-shifting ability, comparing adults with AN with HCs identified five papers employing the Wisconsin Card Sorting Test (WCST) (Heaton et al. 1993), a widely used measure of set-shifting, with a meta-analysis reporting a significant small effect size ( $d=0.36$; Roberts et al. 2007). A further meta-analysis of studies in children and adolescents with AN (Lang et al. 2014), however, found a non-significant pooled effect size $(d=0.20)$. A more recent study comparing children and adolescents with AN with HCs using a larger sample size $(\mathrm{AN}=41, \mathrm{HC}=43)$ found that young people with AN made significantly more perseverative errors than HCs $(d=0.49$; Lang et al. 2015a), suggesting that this cognitive profile is present early on in the disorder before the effects of starvation become prominent. A meta-analysis by $\mathrm{Wu}$ et al. (2014) included a range of neuropsychological tasks assessing setshifting, rather than just the WCST. This revealed a medium effect size for inefficient set-shifting in restrictive AN ( $g=-0.51)$ but no significant effect size for binge/ purge AN. Although the meta-analysis by $\mathrm{Wu}$ et al. (2014) did include studies with children and adolescents, the results did not differentiate between age groups.

Within the ASD literature, five reviews have attempted to synthesize the literature on cognitive flexibility (Hill, 2004; Kenworthy et al. 2008; Sanders et al. 2008; Geurts et al. 2009; Leung \& Zakzanis, 2014). Studies using the WCST have consistently shown difficulties with set-shifting which are stable over time (Hill, 2004); however, this review did not differentiate between studies with adult or child participants and no quantitative synthesis was attempted. A further review (Kenworthy et al. 2008) reports that whilst several studies of cognitive flexibility in children and adults with ASD have demonstrated difficulties relative to $\mathrm{HCs}$, the evidence is inconclusive with two of nine studies using the WCST, one with adults and one with children, producing unclear results. A recent meta-analysis (Leung \& Zakzanis, 2014) comparing neuropsychological performance between patients with ASD and HCs on number of perseverative errors on the computerized WCST, which again pooled both child and adult studies, reported a medium mean effect size $(d=0.68)$. A review of the WCST in ASD (Landry \& Al-Taie, 2016) provided effect sizes for four different WCST outputs including perseveration. In this review, age was found to be negatively correlated with perseveration, the opposite of what has been observed in the AN literature. To our knowledge, however, no reviews have directly compared the set-shifting profiles of children and adults with ASD using the WCST, although, generally, results do suggest that difficulties in set-shifting may be more stable across different age groups, than in AN studies.

Despite the set-shifting difficulties found in people with AN seeming to resemble those of ASD, the cognitive profiles of these two disorders have rarely been directly compared. Oldershaw et al. (2011) statistically compared the neuropsychological profile of currently ill AN patients with published data of individuals with ASD on the WCST and found the two groups to be statistically similar. To our knowledge, this is the only study directly comparing the profiles of the two groups and thus further work is needed to confirm whether the set-shifting profile seen in AN truly resembles that of ASD. Furthermore, the Oldershaw et al. (2011) study only included adult patients. Exploring differences in the set-shifting profiles of children and adults with AN would speak to debates of whether neurocognitive traits such as set-shifting underlie the disorder. Since the publication of the study of Oldershaw et al. (2011), additional studies have been published in both AN and ASD, as demonstrated by the review presented here. As these studies are crosssectional and not longitudinal, inferences on the aetiology of ASD and AN cannot be made but statistically comparing the severity of set-shifting difficulties will aid in the comparison of the neuropsychological profiles of the two disorders.

Difficulties with set-shifting have been implicated in several other psychiatric disorders including schizophrenia (Pantelis et al. 1999); major depression (Austin et al. 1999) and obsessive-compulsive disorder (Leopald \& Backenstrass, 2015), suggesting that this could be a general risk factor for the development of mental disorder, rather than a trait, specific to either AN or ASD. However, evidence of deviant set-shifting in these disorders is less consistent than in $\mathrm{AN}$ and ASD, for example, due to the heterogeneous nature of the disorders. As specific attention has been paid to the shared behavioural traits and intermediate phenotypes in ASD and AN (Treasure, 2013), determining 
both the severity and stability of set-shifting difficulties in the two disorders through meta-analysis is a necessary step to exploring shared underlying mechanisms.

If it is the case that both children and adults with ASD display inefficiencies in set-shifting, it would add evidence to suggest that this trait is stable across the trajectory of the disorder. AN studies have tended to find less difficulty with set-shifting in children than in adults but this finding is difficult to interpret as it may reflect type II errors due to small sample sizes in child studies, with the exception of one study (Lang et al. 2015a). It is therefore unknown whether older and younger people with AN show similar cognitive profiles. The present review aims to use sufficiently powered analysis using pooled previous samples to summarize the literature on the use of the WCST as a measure of set-shifting in both ASD and AN populations and to determine: $(a)$ whether individuals with either ASD or AN have difficulties with setshifting; and $(b)$ whether the severity of these difficulties is comparable in the two disorders in both child and adult populations. This will move us closer to understanding whether the difficulties in set-shifting are stable over the course of AN and will be a necessary but not sufficient step in understanding the relationship between AN and ASD.

\section{Method}

The meta-analyses were conducted according to the 'PRISMA' (preferred reporting items for systematic reviews and meta-analysis) statement (Moher et al. 2009). The quality of each study was assessed using the Clinical Appraisal Skills Programme checklist for case-control studies (Critical Appraisal Skills Programme, 2013). The tool consists of 11 questions, which yield a mixture of 'yes', 'no' and more qualitative answers. In order to calculate an overall quality rating, several questions were split into subquestions and a score of 1 was awarded for every 'yes' answered, with a maximum possible score of 16 . The quality rating for each study is shown in Tables 1 and 2.

\section{Eligibility criteria}

Studies using the WCST and reporting the number or percentage of perseverative errors with a clinical population of either ASD or AN and HCs were included in the review. Due to the heterogeneous nature of ASD and the broadening diagnostic criteria of both ASD and $\mathrm{AN}$ in the Diagnostic and Statistical Manual of Mental Disorders, 5th edition (DSM-V; American Psychiatric Association, 2013), all variants of the two disorders were included.

\section{Information sources and search}

The electronic databases Scopus, PsycINFO, PubMed and Web of Science were searched up to and including January 2016. Search terms included anorexia nervosa OR autism AND set-shifting, Wisconsin, executive function OR cognitive flexibility. Limits included English-language, peer-reviewed articles. Reference lists of eligible papers were also screened, as were existing systematic reviews related of set-shifting in either ASD or AN (Roberts et al. 2007; Lang et al. 2014; Tchanturia, 2015; Landry \& Al-Taie, 2016).

\section{Selection}

The first and principal authors (H.W., K.T.) identified potential titles and screened the abstracts for relevance. Full texts were then retrieved and read to determine eligibility by H.W. Texts deemed eligible were then further screened by K.T. and any papers that did not meet inclusion criteria were excluded. Reasons for exclusion included: the WCST was not used; perseverative errors were not reported; the WCST had been adapted for use with a particular population or there was no $\mathrm{HC}$ group; recovered $\mathrm{AN}$ cases were also excluded.

\section{Data collection and items}

The data items collected from each eligible study were: diagnosis; number of participants; mean age of participants; WCST version; percentage of female participants; how HCs were matched to clinical samples; how co-morbidities were controlled for; diagnostic tools; participant intelligent quotient (IQ); country where the study was conducted; and source of participant recruitment. For AN studies, body mass index (BMI) and illness duration were also extracted. In addition to demographic and experimental paradigm data, the means and standard deviations of perseverative errors on the WCST were used within the meta-analysis.

\section{Risk of bias in individual studies}

The risk of bias in individual studies was assessed by considering how methodology would make an impact on effect size in each study, for example, by attending to how HCs were matched to clinical samples, how the WCST was administered and how participants were recruited.

\section{Summary measure}

The principal summary measure used for analysis from all studies was the difference in means and standard deviations of perseverative errors or percentage of 
Table 1. Demographic information for AN studies

\begin{tabular}{|c|c|c|c|c|c|c|c|c|c|c|c|c|c|}
\hline Author/date & Group & $n$ & $\begin{array}{l}\text { Mean age, } \\
\text { years (S.D.) }\end{array}$ & $\begin{array}{l}\text { Mean BMI, } \\
\mathrm{kg} / \mathrm{m}^{2} \text { (S.D.) }\end{array}$ & $\begin{array}{l}\text { Mean illness } \\
\text { duration, } \\
\text { years (s.D.) }\end{array}$ & $\begin{array}{l}\text { Mean IQ } \\
\text { (s.D.) }\end{array}$ & $\begin{array}{l}\text { HCs } \\
\text { matched } \\
\text { by }\end{array}$ & WCST version & $\begin{array}{l}\% \\
\text { Female } \\
\text { AN }\end{array}$ & $\begin{array}{l}\% \\
\text { Female } \\
\text { HC }\end{array}$ & Recruited from & $\begin{array}{l}\text { Diagnostic } \\
\text { tool }\end{array}$ & $\begin{array}{l}\text { Quality } \\
\text { score }\end{array}$ \\
\hline \multicolumn{14}{|l|}{ Adult studies } \\
\hline \multirow[t]{2}{*}{$\begin{array}{r}\text { Abbate-Daga } \\
\text { et al. (2011) }\end{array}$} & AN-R & 30 & $24.1(6.7)$ & $15.6(1.6)$ & $5.2(4.2)$ & & Age & Paper version & 100 & 100 & $\begin{array}{l}\text { ED service, } \\
\text { mixed treatment }\end{array}$ & SCID & 13 \\
\hline & $\mathrm{HC}$ & 30 & $24.7(2.6)$ & $21(2.2)$ & & & & & & & University & & \\
\hline \multirow[t]{2}{*}{$\begin{array}{l}\text { Abbate-Daga } \\
\text { et al. }(2014)\end{array}$} & AN & 94 & $24.7(7.3)$ & $15.2(2.0)$ & & & Race & $\begin{array}{l}128 \text { cards, } \\
\text { paper version }\end{array}$ & 100 & 100 & $\begin{array}{l}\text { ED service, } \\
\text { mixed treatment }\end{array}$ & SCID & 11 \\
\hline & $\mathrm{HC}$ & 59 & $25.1(3.2)$ & $20.6(2.0)$ & $7.1(6.6)$ & & & & & & University & & \\
\hline \multirow{2}{*}{$\begin{array}{l}\text { Aloi et al. } \\
\text { (2015) }\end{array}$} & AN-R & 45 & $22.8(5.6)$ & $15.5(1.4)$ & & & & Italian version & 100 & & ED service & SCID & 13 \\
\hline & $\mathrm{HC}$ & 45 & $25.6(3.5)$ & $20.2(1.6)$ & & & & & & & University & & \\
\hline \multirow{2}{*}{$\begin{array}{l}\text { Fagundo et al. } \\
\quad \text { (2012) }\end{array}$} & $\mathrm{AN}$ & 35 & $28.1(8.2)$ & $17.2(1.4)$ & & & & 128 cards & 100 & & ED service & SCID & 13 \\
\hline & $\mathrm{HC}$ & 137 & $24.8(7.0)$ & $21.5(2.7)$ & & & & & & & University & & \\
\hline \multirow[t]{2}{*}{$\begin{array}{l}\text { Fassino et al. } \\
\quad(2002)\end{array}$} & AN-R & 20 & $23.8(6.6)$ & $15.2(2.2)$ & & & Gender & $\begin{array}{l}\text { Nelson's } \\
\text { version, } 48 \\
\text { cards }\end{array}$ & 100 & 100 & ED service & SCID & 13 \\
\hline & $\mathrm{HC}$ & 20 & $23.1(2.9)$ & $20.6(1.7)$ & & & & & & & High school, unive & rsity & \\
\hline \multirow[t]{2}{*}{$\begin{array}{l}\text { Galimberti } \\
\text { et al. (2013) }\end{array}$} & AN & 29 & $24.1(6.8)$ & $16.2(4.0)$ & $6(5.1)$ & & Gender & Computerized & 100 & 100 & $\begin{array}{l}\text { Psychiatric } \\
\text { hospital }\end{array}$ & DSM-IV-TR & 13 \\
\hline & $\mathrm{HC}$ & 29 & $28.6(11.9)$ & & & & & & & & Local community & & \\
\hline \multirow[t]{2}{*}{$\begin{array}{c}\text { Giel et al. } \\
\text { (2012) }\end{array}$} & AN & 15 & $23.9(5.7)$ & $15.8(1.9)$ & & $103.0(1.0)^{\mathrm{a}}$ & $\begin{array}{l}\text { Age, gender, } \\
\text { education }\end{array}$ & Computerized & 100 & 100 & $\begin{array}{l}\text { ED service, } \\
\text { mixed treatment }\end{array}$ & SCID & 15 \\
\hline & $\mathrm{HC}$ & 35 & $30.2(10.9)$ & $22.0(1.9)$ & & $\begin{array}{l}110.0 \\
(17.0)^{\mathrm{a}}\end{array}$ & & & & & Local community & & \\
\hline \multirow[t]{2}{*}{$\begin{array}{l}\text { Goddard et al. } \\
\text { (2014) }\end{array}$} & AN & 29 & $26.2(8.2)$ & $17.7(2.2)$ & & $106.9(9.4)$ & & Computerized & 0 & 0 & $\begin{array}{l}\text { ED service, } \\
\text { mixed treatment }\end{array}$ & EDE & 15 \\
\hline & $\mathrm{HC}$ & 42 & $26.4(7.2)$ & $23.2(2.5)$ & & $108.4(8.2)$ & & & & & University & & \\
\hline \multirow[t]{2}{*}{$\begin{array}{l}\text { Harrison et al. } \\
\quad(2012)\end{array}$} & AN & 50 & $27.1(9.3)$ & $15.4(1.8)$ & $9.2(9.8)$ & $111.4(8.7)$ & & Computerized & 100 & 100 & $\begin{array}{l}\text { ED service, } \\
\text { mixed treatment }\end{array}$ & DSM-V & 16 \\
\hline & $\mathrm{HC}$ & 89 & $28.5(9.9)$ & $21.6(1.9)$ & & $112.3(7.4)$ & & & & & University & & \\
\hline \multirow{2}{*}{$\begin{array}{l}\text { Koba et al. } \\
\quad(2002)\end{array}$} & AN & 11 & & & & & & & & & & & \\
\hline & $\mathrm{HC}$ & 7 & & & & & & & & & & & \\
\hline $\begin{array}{l}\text { Nakazato et al. } \\
\quad(2010)\end{array}$ & AN & 27 & $27.7(10.6)$ & $15.4(1.9)$ & $10(10.6)$ & & Age & $\begin{array}{l}\text { Computerized, } \\
\text { 4th edition }\end{array}$ & 100 & 100 & $\begin{array}{l}\text { ED service, } \\
\text { mixed treatment }\end{array}$ & DSM-IV & 13 \\
\hline
\end{tabular}




\begin{tabular}{|c|c|c|c|c|c|}
\hline \multirow[b]{2}{*}{$\begin{array}{l}\text { Ohrmann et al. } \\
\text { (2004) }\end{array}$} & $\mathrm{HC}$ & 28 & $26.9(5.8)$ & $22.3(2.5)$ & \multirow[b]{2}{*}{$5.5(5.2)$} \\
\hline & AN & 11 & $22.7(3.8)$ & $15.2(1.2)$ & \\
\hline \multirow{3}{*}{$\begin{array}{l}\text { Pignatti \& } \\
\text { Bernasconi } \\
(2013)\end{array}$} & $\mathrm{HC}$ & 11 & $27.5(6.3)$ & $21.3(2.1)$ & \\
\hline & AN & 23 & $29.1(7.4)$ & $16.0(2.0)$ & \\
\hline & $\mathrm{HC}$ & 20 & $27.8(7.0)$ & $21.6(1.4)$ & \\
\hline \multirow{2}{*}{$\begin{array}{l}\text { Sato et al. } \\
\text { (2013) }\end{array}$} & AN & 15 & $23(7.0)$ & $14.6(1.5)$ & $3.6(3.7)$ \\
\hline & $\mathrm{HC}$ & 15 & $22(3.0)$ & $20.6(1.2)$ & \\
\hline \multirow{2}{*}{$\begin{array}{l}\text { Steinglass et al. } \\
\quad(2006)\end{array}$} & AN & 15 & $25.6(6.0)$ & $19(1.0)$ & $10.8(5.8)$ \\
\hline & $\mathrm{HC}$ & 11 & $24(3.1)$ & $22.1(1.8)$ & \\
\hline \multirow{2}{*}{$\begin{array}{l}\text { Tchanturia } \\
\text { et al. (2012) }\end{array}$} & AN & 171 & $25.4(8.2)$ & $15.2(1.9)$ & \\
\hline & $\mathrm{HC}$ & 199 & $27.7(8.8)$ & $21.9(1.9)$ & \\
\hline \multirow{2}{*}{$\begin{array}{c}\text { Thompson } \\
\text { (1993) }\end{array}$} & AN & 10 & 25.8 & & \\
\hline & $\mathrm{HC}$ & 10 & 23.2 & & \\
\hline \multirow{3}{*}{$\begin{array}{l}\text { Van Autreve } \\
\text { et al. (2013) }\end{array}$} & AN-R & 31 & $26(12.0)$ & $14.4(2.0)$ & \\
\hline & AN-BP & 20 & $20(4.0)$ & $16.4(1.1)$ & \\
\hline & $\mathrm{HC}$ & 26 & $19.0(2.0)$ & 20.9 (1.8) & \\
\hline \multicolumn{6}{|c|}{ Child/adolescent studies } \\
\hline \multirow{2}{*}{$\begin{array}{l}\text { Andres- } \\
\text { Perpina et al. } \\
(2011)\end{array}$} & AN & 37 & $15.4(1.5)$ & $15.3(1.4)$ & $13.2(10.7)^{\mathrm{b}}$ \\
\hline & $\mathrm{HC}$ & 41 & $15.4(1.5)$ & & \\
\hline \multirow{2}{*}{$\begin{array}{l}\text { Fitzpatrick } \\
\text { et al. (2012) }\end{array}$} & AN & 32 & $14.9(1.9)$ & $78.1(6.6)^{b}$ & \\
\hline & $\mathrm{HC}$ & 22 & $15.4(1.9)$ & $105.7(12.8)^{\mathrm{b}}$ & \\
\hline $\begin{array}{l}\text { Lang et al. } \\
\qquad(2015 a)\end{array}$ & AN & 41 & $15.1(1.8)$ & $16.2(1.5)$ & \\
\hline $\begin{array}{l}\text { McAnarney } \\
\text { et al. }(2011)\end{array}$ & AN-R & 24 & $16.3(1.2)$ & $16.7(1.3)$ & \\
\hline
\end{tabular}

\begin{tabular}{|c|c|c|c|c|c|c|c|}
\hline & & & & & Local community & & \\
\hline & Age, gender & $\begin{array}{l}\text { Modified } \\
\text { WCST }\end{array}$ & 100 & 100 & $\begin{array}{l}\text { Psychiatric } \\
\text { in-patient } \\
\text { service }\end{array}$ & SCID & 11 \\
\hline & $\begin{array}{l}\text { Age, } \\
\text { education }\end{array}$ & Italian version & 100 & 100 & ED service & DSM-IV-TR & 11 \\
\hline $97.8(13.7)$ & Age, IQ & $\begin{array}{l}\text { Computerized } \\
128 \text { trials }\end{array}$ & 100 & 100 & $\begin{array}{l}\text { ED service, } \\
\text { mixed treatment }\end{array}$ & DSM-IV-TR & 15 \\
\hline 104.9 (11.3) & & & & & & & \\
\hline $109.8(12.1)$ & Gender & $\begin{array}{l}\text { Computerized, } \\
\text { 4th edition }\end{array}$ & 100 & 100 & $\begin{array}{l}\text { ED research } \\
\text { clinic }\end{array}$ & SCID & 10 \\
\hline $115.5(6.6)$ & & & & & & & \\
\hline & Age & $\begin{array}{l}\text { Computerized, } \\
\text { 4th edition }\end{array}$ & 100 & 100 & $\begin{array}{l}\text { ED service, } \\
\text { mixed treatment }\end{array}$ & DSM-IV & 12 \\
\hline 115.8 & $\begin{array}{l}\text { Age, education } \\
\text { location }\end{array}$ & , IQ, class, & 100 & 100 & ED out-patient & DSM-III-R & 12 \\
\hline 119.7 & & & & & Local community & & \\
\hline & Gender & $\begin{array}{l}\text { Computerized, } \\
\text { 4th edition }\end{array}$ & 100 & 100 & $\begin{array}{l}\text { ED service, } \\
\text { mixed treatment } \\
\text { University }\end{array}$ & DSM-IV & 12 \\
\hline & Age & & 94.6 & 90.2 & $\begin{array}{l}\text { Psychiatric } \\
\text { hospital }\end{array}$ & DSM-IV-TR & 12 \\
\hline 119.5 (14.5) & & $\begin{array}{l}\text { Computerized, } \\
\text { 4th edition }\end{array}$ & 100 & 100 & $\begin{array}{l}\text { Local school } \\
\text { ED in-patient }\end{array}$ & DSM-IV & 12 \\
\hline 119.5 (11.0) & & & & & Local community & & \\
\hline $107.1(13.8)$ & Gender, age & $\begin{array}{l}\text { Computerized, } \\
\text { 4th edition }\end{array}$ & 100 & 100 & $\begin{array}{l}\text { ED service, } \\
\text { mixed treatment }\end{array}$ & $\begin{array}{l}\text { SCID, } \\
\text { DSM-V }\end{array}$ & 15 \\
\hline $111.6(10.7)$ & $\begin{array}{l}\text { Age, SES, IQ, } \\
\text { ethnicity }\end{array}$ & Computerized & 100 & 100 & & DSM-IV & 9 \\
\hline
\end{tabular}




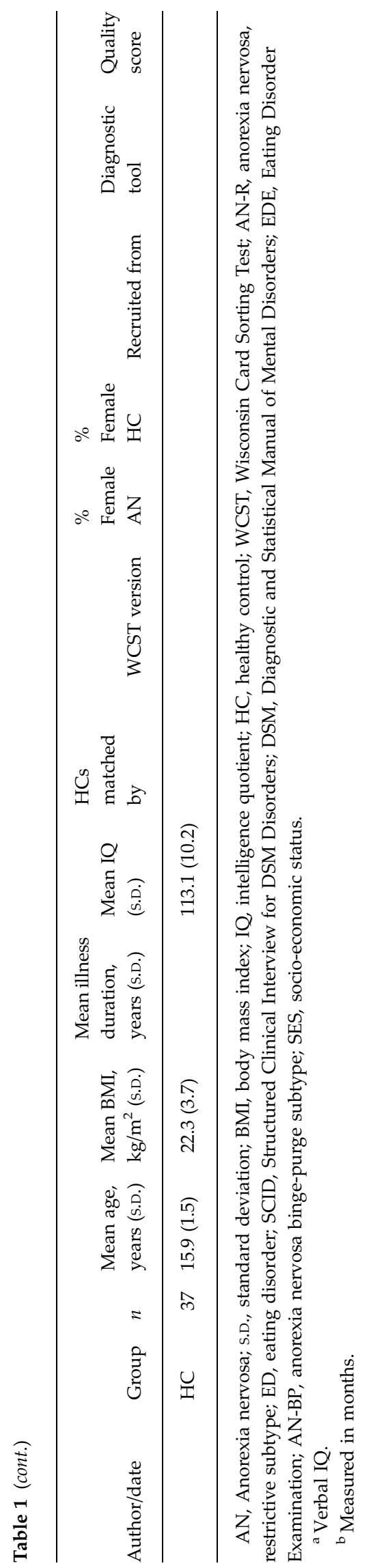

perseverative errors between the clinical sample (ASD or AN) and HCs on the WCST. Standardized mean scores were used within the analysis to account for the variation in version of the WCST used in the studies. The WCST involves sorting cards into one of four categories according to one of three sorting rules: colour, shape or number. Once a card has been sorted, the participant is given feedback as to whether they sorted the card according to the correct rule. The paradigm yields a number of different outputs which can be analysed including perseverative errors, when the participant continues to sort cards according to a previous rule (Lezak, 1995). The WCST is a widely used measure of general executive function (Heaton et al. 1993; Greve et al. 2005). The WCST is assumed to consist of three factors: perseveration, failure to maintain set and idiosyncratic sorting (Greve et al. 1998). An increased number of perseverative errors has been associated with frontal lobe dysfunction (Milner, 1963; Nelson, 1976) and, as a robust measure of conceptual flexibility, was therefore the main focus for this review.

Only studies using the WCST, rather than other setshifting paradigms, were included because this is a widely used measure across a wide range of disorders, believed to reliably assess cognitive flexibility (Holmén et al. 2012). There is less variability in terms of test properties (64- or 128-card version, computerized or pen-paper) compared with other paradigms such as the trail-making test (Atkinson \& Ryan, 2008). Unlike other paradigms, the WCST also provides numerous different outputs, including perseveration, the most relevant measure in relation to set-shifting. It is widely used in both AN and ASD research allowing for direct comparison between these groups. The WCST is also considered to be a purer measure of set-shifting, compared with other paradigms which may tap into other cognitive domains (Steinglass et al. 2006).

\section{Synthesis of data}

Synthesis of results for meta-analysis meant that only studies reporting mean scores of perseverative errors were included in the analysis. Therefore, studies which reported standardized rather than raw scores or presented results visually were not included. The reasons studies were excluded are presented in Fig. 1 . The meta-analyses were performed by pooling the standardized effect sizes of each study using a random-effects model. Random-effects models assume that as well as within-group variability caused by sampling variability of scores, mean effect size is also caused by differences between studies (Borenstein et al. 2009). If the between-study heterogeneity is present, the random-effects model results in estimates 
Table 2. Demographic information for ASD studies

\begin{tabular}{|c|c|c|c|c|c|c|c|c|c|c|c|}
\hline Author/date & Group & $n$ & $\begin{array}{l}\text { Mean age, } \\
\text { years (s.D.) }\end{array}$ & $\begin{array}{l}\text { Mean IQ } \\
\text { (s.D.) }\end{array}$ & HCs matched by & WCST version & $\begin{array}{l}\% \text { Female } \\
\text { ASD }\end{array}$ & $\begin{array}{l}\% \text { Female } \\
\mathrm{HC}\end{array}$ & Recruited from & $\begin{array}{l}\text { Diagnostic } \\
\text { tool }\end{array}$ & $\begin{array}{l}\text { Quality } \\
\text { score }\end{array}$ \\
\hline \multicolumn{12}{|l|}{ Adult studies } \\
\hline \multirow[t]{2}{*}{$\begin{array}{l}\text { Ambery et al. } \\
\text { (2006) }\end{array}$} & AS & 27 & $37.6(14.6)$ & & VIQ and PIQ & 128 cards & 18.5 & 20 & $\begin{array}{l}\text { Specialist assessment } \\
\text { service }\end{array}$ & $\begin{array}{l}\text { ICD-10, ADI, } \\
\text { ADOS }\end{array}$ & 13 \\
\hline & $\mathrm{HC}$ & 20 & $33.5(12.0)$ & & & & & & & & \\
\hline \multirow[t]{2}{*}{ Eack et al. (2013) } & ASD & 43 & $24.9(5.8)$ & & & & 12 & 33 & Out-patient service & ADOS & 14 \\
\hline & $\mathrm{HC}$ & 24 & $26.3(5.5)$ & & & & & & & & \\
\hline \multirow{2}{*}{$\begin{array}{l}\text { Goldstein } \\
\text { et al. }(2001)\end{array}$} & HFA & 103 & $18.2(10.1)$ & & FIQ and VIQ & 128 cards & 13.6 & 10.7 & & ADI, ADOS & 12 \\
\hline & $\mathrm{HC}$ & 103 & $19.0(10.1)$ & & & & & & & & \\
\hline \multirow[t]{2}{*}{$\begin{array}{l}\text { Griebling } \\
\text { et al. }(2010)\end{array}$} & HFA & 38 & $17.9(10.0)$ & & FIQ, PIQ and VIQ & & 5.3 & 4.2 & $\begin{array}{l}\text { University recruitment } \\
\text { programme }\end{array}$ & $\begin{array}{l}\text { ADI, ADOS, } \\
\text { clinical } \\
\text { description }\end{array}$ & 10 \\
\hline & $\mathrm{HC}$ & 40 & $18.6(9)$ & & & & & & $\begin{array}{l}\text { University recruitment } \\
\text { programme }\end{array}$ & & \\
\hline \multirow[t]{2}{*}{ Hill \& Bird (2006) } & AS & 22 & $31.1(13.1)$ & & $\begin{array}{l}\text { Age, gender, general } \\
\text { ability }\end{array}$ & $\begin{array}{c}\text { Nelson's } \\
\text { version }\end{array}$ & 22.3 & 36.4 & Support groups & APA criteria & 11 \\
\hline & $\mathrm{HC}$ & 22 & $33.5(14.5)$ & & & & & & $\begin{array}{l}\text { University recruitment } \\
\text { programme }\end{array}$ & & \\
\hline \multirow[t]{2}{*}{ Lopez et al. (2005) } & AS & 17 & 29 & & & & 17.6 & 35.3 & & $\begin{array}{l}\text { ADI, ADOS, } \\
\text { GARS }\end{array}$ & 10 \\
\hline & $\mathrm{HC}$ & 17 & 29 & & & & & & & & \\
\hline \multirow[t]{2}{*}{ Maes et al. (2011) } & ASD & 17 & $45.2(13.1)$ & & & Computerized & 23.5 & 21.1 & $\begin{array}{l}\text { University medical } \\
\text { centre }\end{array}$ & & 13 \\
\hline & $\mathrm{HC}$ & 19 & $38.4(14.8)$ & & & & & & University & & \\
\hline \multirow[t]{2}{*}{ Rumsey (1985) } & AS & 9 & $27(7)$ & & $\begin{array}{l}\text { Highest level of } \\
\text { education }\end{array}$ & 128 cards & 0 & 0 & Nationwide register & DSM-III & 11 \\
\hline & $\mathrm{HC}$ & 10 & $28(5)$ & & & & & & & & \\
\hline \multirow[t]{2}{*}{$\begin{array}{l}\text { Sumiyoshi } \\
\text { et al. (2011) }\end{array}$} & ASD & 22 & $24.5(4.0)$ & & Age & $\begin{array}{l}\text { Computerized } \\
64 \text { cards }\end{array}$ & 13.6 & 26.7 & Out-patient service & $\begin{array}{l}\text { DSM-IV, AQ, } \\
\text { CARS }\end{array}$ & 13 \\
\hline & $\mathrm{HC}$ & 15 & $29.7(6.4)$ & & & & & & $\begin{array}{l}\text { Word of mouth and } \\
\text { college }\end{array}$ & & \\
\hline \multirow[t]{2}{*}{$\begin{array}{l}\text { Yasuda et al. } \\
\text { (2014) }\end{array}$} & ASD & 33 & $26.1(11.5)$ & & $\begin{array}{l}\text { Age, gender, education, } \\
\text { FIQ, PIQ, VIQ }\end{array}$ & $\begin{array}{l}\text { Keio version, } \\
48 \text { cards }\end{array}$ & 39.4 & 39.4 & $\begin{array}{l}\text { Out-patient and } \\
\text { in-patient services }\end{array}$ & $\begin{array}{l}\text { DSM-IV-TR, } \\
\text { ADI-R, PARS, } \\
\text { AQ-J }\end{array}$ & 13 \\
\hline & $\mathrm{HC}$ & 33 & $26.8(9.9)$ & & & & & & & & \\
\hline
\end{tabular}




\begin{tabular}{|c|c|c|c|c|c|c|c|c|c|c|c|}
\hline Author/date & Group & $n$ & $\begin{array}{l}\text { Mean age, } \\
\text { years (S.D.) }\end{array}$ & $\begin{array}{l}\text { Mean IQ } \\
\text { (s.D.) }\end{array}$ & HCs matched by & WCST version & $\begin{array}{l}\% \text { Female } \\
\text { ASD }\end{array}$ & $\begin{array}{l}\% \text { Female } \\
\mathrm{HC}\end{array}$ & Recruited from & $\begin{array}{l}\text { Diagnostic } \\
\text { tool }\end{array}$ & $\begin{array}{l}\text { Quality } \\
\text { score }\end{array}$ \\
\hline \multicolumn{12}{|c|}{ Adolescent/adult studies } \\
\hline \multirow[t]{2}{*}{$\begin{array}{l}\text { Minshew } \\
\text { et al. (1992) }\end{array}$} & AS & 15 & $21.1(8.0)$ & $95.7(13.6)$ & $\begin{array}{l}\text { Age, FIQ, gender and } \\
\text { race }\end{array}$ & & 0 & 0 & & ADI, ADOS & 10 \\
\hline & $\mathrm{HC}$ & 15 & $21.3(8.3)$ & $96.5(12.3)$ & & & & & & & \\
\hline \multirow[t]{2}{*}{$\begin{array}{l}\text { Minshew } \\
\text { et al. (1997) }\end{array}$} & AS & 33 & $20.9(9.7)$ & $100.9(13.0)$ & & & 12.2 & 12.2 & & $\begin{array}{l}\text { ADI, ADOS, } \\
\text { clinical } \\
\text { description }\end{array}$ & 12 \\
\hline & $\mathrm{HC}$ & 33 & $21.2(10.0)$ & $100.5(11.7)$ & & & & & & & \\
\hline \multirow[t]{2}{*}{$\begin{array}{l}\text { Minshew } \\
\text { et al. (2002) }\end{array}$} & HFA & 90 & $21.4(9.7)$ & $98.0(16.0)$ & $\begin{array}{l}\text { Age, FIQ, gender and } \\
\text { race }\end{array}$ & 128 cards & & & & $\begin{array}{l}\text { ADI, ADOS, } \\
\text { clinical } \\
\text { description }\end{array}$ & 10 \\
\hline & $\mathrm{HC}$ & 107 & $21.2(9.8)$ & $100.9(13.4)$ & & & & & & & \\
\hline \multicolumn{12}{|l|}{ Child studies } \\
\hline \multirow[t]{2}{*}{ Kado et al. (2012) } & PDD & 52 & $118.5(29.0)^{\mathrm{a}}$ & $97.7(10.1)$ & & $\begin{array}{l}\text { Keio version, } \\
48 \text { cards }\end{array}$ & 25 & 21.2 & & DSM-IV & 10 \\
\hline & $\mathrm{HC}$ & 52 & $119.7(33.5)^{\mathrm{a}}$ & & & & & & & & \\
\hline \multirow[t]{2}{*}{$\begin{array}{l}\text { Kaland et al. } \\
\quad(2008)\end{array}$} & AS/HFA & 13 & $16.4(2.8)$ & $109(11.5)$ & IQ and age & $\begin{array}{l}\text { Computerized } \\
128 \text { cards }\end{array}$ & 0 & 0 & Specialist school & $\begin{array}{l}\text { ICD-10, ADI-R, } \\
\text { ADOS }\end{array}$ & 12 \\
\hline & $\mathrm{HC}$ & 13 & $15.6(3.1)$ & $109.6(10.4)$ & & & & & School & & \\
\hline \multirow[t]{2}{*}{$\begin{array}{l}\text { Lam \& } \\
\quad \text { Yeung (2012) }\end{array}$} & ASD & 12 & $6.1(8.2)$ & & Gender, age, VIP, PIQ & & & & Child ASD service & & 10 \\
\hline & $\mathrm{HC}$ & 12 & $5.6(1.0)$ & & & & & & & & \\
\hline \multirow[t]{2}{*}{ Li et al. (2014) } & HFA & 38 & $9.6(2.3)$ & $109.8(18.9)$ & Age and gender & Computerized & 21.1 & 21.1 & Specialist service & $\begin{array}{l}\text { DSM-V, ABC, } \\
\text { CARS, AQ }\end{array}$ & 8 \\
\hline & $\mathrm{HC}$ & 31 & $10.2(1.5)$ & $113.0(15.1)$ & & & & & & & \\
\hline \multirow[t]{2}{*}{$\begin{array}{l}\text { Pascualvaca } \\
\text { et al. (1998) }\end{array}$} & ASD & 23 & $8.7(1.1)$ & $77.6(14.5)$ & PIQ & $\begin{array}{l}\text { Manual } 128 \\
\text { cards }\end{array}$ & 34.8 & 34.8 & Autism society & $\begin{array}{l}\text { DSM-III-R, } \\
\text { CARS }\end{array}$ & 15 \\
\hline & $\mathrm{HC}$ & 23 & $6.7(1.8)$ & $110.2(10.2)$ & & & & & School & & \\
\hline \multirow[t]{2}{*}{$\begin{array}{l}\text { Pooragha } \\
\text { et al. (2013) }\end{array}$} & HFA & 15 & $9.3(1.8)$ & $92.7(7.01)$ & Age and education & & 6.7 & 15.4 & Hospital & & 10 \\
\hline & $\mathrm{HC}$ & 15 & $10.1(2.4)$ & $101.1(9.1)$ & & & & & & & \\
\hline \multirow[t]{2}{*}{ Shu et al. (2001) } & AS & 26 & & $80.0(12.0)$ & Age & $\begin{array}{l}\text { Computerized } \\
128 \text { cards }\end{array}$ & & & Autism society & DSM-III-R & 14 \\
\hline & $\mathrm{HC}$ & 52 & & & & & & & School & & \\
\hline $\begin{array}{l}\text { Tsuchiya } \\
\text { et al. (2005) }\end{array}$ & HFA & 17 & $12.5(4.3)$ & $92.3(7.5)$ & Age & $\begin{array}{l}\text { Computerized } \\
128 \text { cards }\end{array}$ & 5.9 & 48 & Paediatric department & & 10 \\
\hline
\end{tabular}




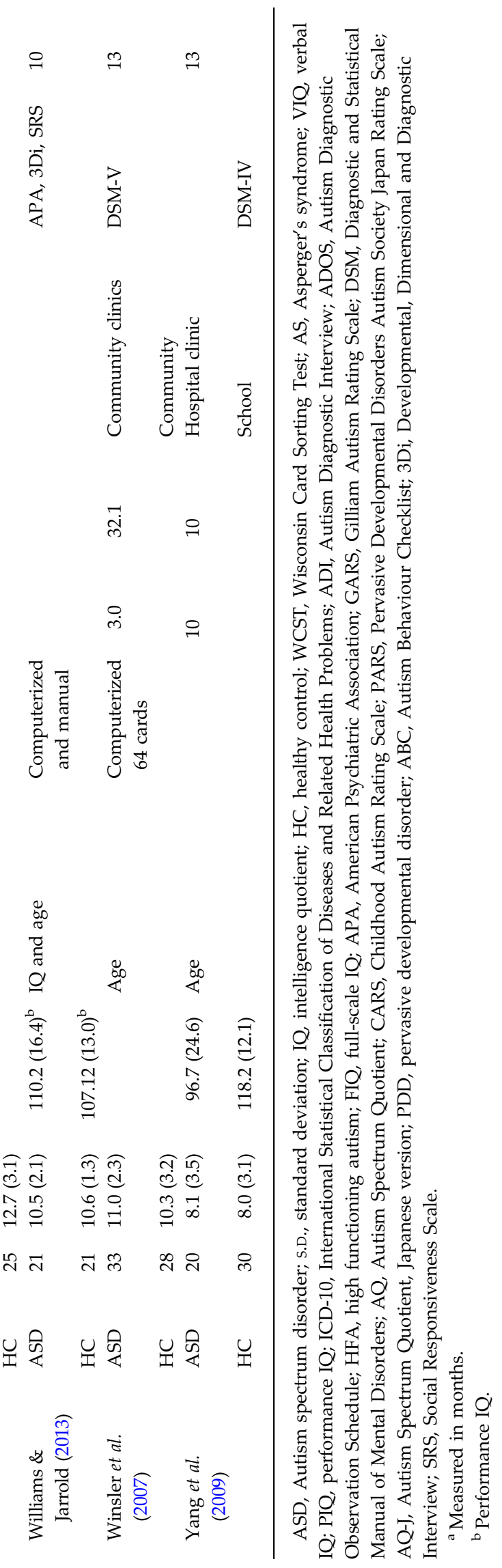

with wider confidence intervals (CIs) than fixed-effects models.

\section{Statistical analysis}

Analysis was carried out in STATA 13 (StataCorp, USA) with the following user contributed commands metan (Bradburn et al. 1998), metabias, metatrim (Steichen, 1998) and metareg (Sharp, 1998). Cohen's $d$ was used to estimate the effect sizes and is reported for all studies together with $95 \%$ CIs. The effect sizes were interpreted according to Cohen's (1988) definitions of small $(\geqslant 0.20 \leqslant 0.50)$, medium $(\geqslant 0.50$ $\leqslant 0.80)$, large $(\geqslant 0.80 \leqslant 1.30)$ and very large $(\geqslant 1.30)$. Positive effect size indicates that the clinical group (ASD or AN) made more perseverative errors than HCs. A $p$ value of $<0.05$ indicates significant difference between groups.

\section{Risk of bias across studies}

Publication bias was assessed by visually evaluating funnel plots of each study's standardized mean differences against its precision and formally by Egger's test (Egger et al. 1997) to see if a study's precision was related to effect size (Borenstein et al. 2009).

\section{Additional analysis}

Between-study heterogeneity was measured by calculating $I^{2}$ (Higgins et al. 2003) based on Cochran's $Q$ test: measure of heterogeneity, $I^{2}=100 \% \times(Q-\mathrm{df}) / Q$, where $\mathrm{df}$ is degrees of freedom. $I^{2}$ ranges between $0 \%$, indicating no heterogeneity and $100 \%$, indicating high heterogeneity. Child/adolescent studies were analysed separately as well as overall effect size being calculated to explore whether effect was related to participant age. In addition, meta-regression was used to test for differences between ASD and AN participants using diagnosis (AN or ASD) as the moderator.

\section{Results}

\section{Study selection}

In all, 24 studies in ASD and 22 in AN were included in the meta-analyses consisting of a total of 815 AN participants matched to $956 \mathrm{HCs}$ and 739 ASD participants matched to $760 \mathrm{HCs}$. The selection process for both AN and ASD studies is shown in Fig. 1.

\section{Study characteristics}

All extracted information for AN and ASD studies included in the systematic reviews and meta-analyses is presented in Tables 1 and 2, respectively. Quality 

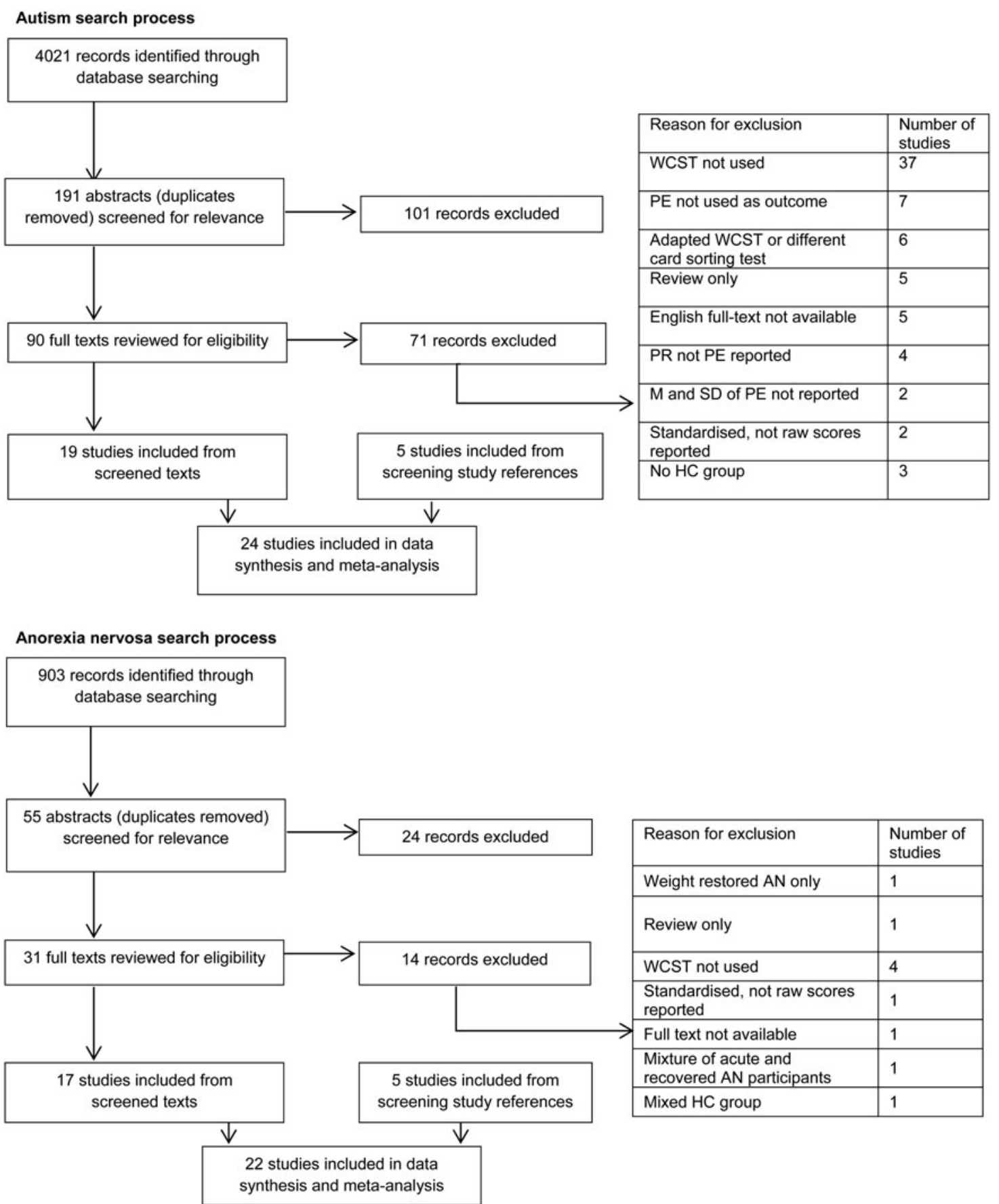

Fig. 1. Systematic review search process. WCST, Wisconsin Card Sorting Test; PE, perseverative error; PR, perseverative response; $\mathrm{M}$, mean; $\mathrm{SD}$, standard deviation; $\mathrm{HC}$, healthy control; $\mathrm{AN}$, anorexia nervosa.

ratings for each study ranged from 16 (highest possible score) to 8 ( $\mathrm{Li}$ et al. 2014). This study included very little information on the recruitment of clinical participants or HCs but the results were sufficiently detailed for it to be included in the current review. Of the AN studies, the lowest score was 9 (McAnarney et al. 2011). This was presented as a brief report and thus methodological detail was missing. The results of this study were also unclear so the authors were contacted by K.T. to clarify before data were included in the meta-analysis. The full version of the paper by Koba et al. (2002) was unavailable to the authors at the time of this review; however, as it has been included in previous reviews (Roberts et al. 2007) it was included in the current analysis.

One ASD study (Goldstein et al. 2001) included both child and adult participants. Although the authors did split the ASD participants into 'old' and 'young' for 
analysis, the control group was not split so the pooled results of children and adults, with a mean age of 18 years, were included in the adult meta-analysis. The majority of ASD studies reported IQ which ranged from 77 to 113.1 in the ASD group and 89 to 118 in $\mathrm{HCs}$, indicating no intellectual disability. Nine AN studies also reported IQ, all of which fell within the normal range (97.8-119.5 for AN and 104.9-119.7 for HCs). Of the AN studies, all but two included only female participants, whereas one study included only males (Goddard et al. 2014) and one included over $90 \%$ of females (Andres-Perpina et al. 2011). In the ASD studies three studies included only males, with the other studies ranging from 10 to $48 \%$ female participants.

Within both clinical groups, diagnosis varied. For example, within the ASD studies eight papers reported using a diagnosis of ASD, seven used high functioning autism, seven used Asperger's syndrome and one used pervasive developmental disorder. In the AN studies, 17 used a diagnosis of AN while five only included participants with restrictive subtype $\mathrm{AN}$, one of which had a separate binge-purge subtype AN group. Data on mean BMI were also extracted from AN studies and ranged from 14.4 to 19.0 in the AN group. In the ASD studies, diagnosis was given using a variety of clinical measures; eight used both the Autism Diagnostic Interview and the Autism Diagnostic Observation Schedule; 12 used diagnostic criteria taken from the DSM and several self-report measures including the Childhood Autism Rating Scale, the Autism Spectrum Quotient and the Social Responsiveness Scale were also used. In the AN studies, all studies used diagnostic criteria from the DSM, either assessed directly or by using the Structured Clinical Interview for DSM Disorders (SCID; American Psychiatric Association, 2013). One study used the Eating Disorder Examination (Fairburn \& Cooper, 1993). Several ASD and AN studies excluded HCs who had a history of psychiatric disorder but few studies reported co-morbidities, although several AN studies assessed for depression, anxiety and/or obsessive-compulsive disorder.

Sources of participant recruitment also differed across studies; in AN studies, 10 studies recruited their AN sample from specialist eating disorder services consisting of a mixture of out-, day- and inpatient treatment. Four studies did not state what type of treatment the participants were receiving; one recruited from an out-patient service; one from an inpatient service and three from psychiatric hospital settings. HCs were recruited from universities, schools or the local community. In nine of the ASD studies, ASD participants were recruited from a variety of specialist, out-patient or in-patient services for people with ASD; four from autism societies, national registers or research recruitment programmes and one from a specialist school. HCs were recruited from recruitment programmes, schools or the community. Of the ASD studies, 10 were conducted in the USA; four in Japan; three in the UK; two in China and one in the Netherlands, Denmark, Hong Kong, Iran and Taiwan, respectively. Six of the AN studies were conducted in Italy; six in the UK; three in the USA; two in Germany, Japan and Spain and one in Belgium.

\section{Risk of bias}

There was good symmetry within all funnel plots indicating no relationship between effect and study size and thus no publication bias. Egger's test indicated no evidence of publication bias ( $\left.p^{\prime} \mathrm{s}>0.21\right)$. Analysis for correction of publication bias (trim-and-fill method) estimated two missing studies but this did not change the effect size considerably, so uncorrected data are presented here.

\section{Synthesis of results}

The forest plot of AN studies included in the meta-analysis is shown in Fig. 2. The random-effects analysis with a total sample size of 1731 ( $\mathrm{AN}=815$, $\mathrm{HC}=916$ ) revealed a significant difference between $\mathrm{AN}$ and $\mathrm{HC}$ groups on the number of perseverative errors on the WCST $(d=0.43,95 \%$ CI $0.26-0.61) z=4.83$, $p \leqslant 0.001$ ). When adult AN studies were analysed separately, there was a significant difference between AN and HC groups with a medium effect size $(d=0.48$, 95\% CI 0.28-0.68, $z=4.70, p \leqslant 0.001)$. When the four child and adolescent studies were analysed separately, there was no significant difference between $\mathrm{AN}$ and HC groups $(d=0.25,95 \% \mathrm{CI}-0.05$ to $0.55, z=1.66$, $p=0.096)$.

The forest plot of ASD studies included in the metaanalysis is displayed in Fig. 3. The random-effects analysis with a total sample size of 1499 (ASD $=739$, $\mathrm{HC}=760$ ) revealed a significant difference between ASD and HC groups on the number of perseverative errors obtained during the WCST $(d=0.65,95 \% \mathrm{CI}$ $0.51-0.80, z=9.91, p \leqslant 0.001)$. For adult studies, there was a medium effect size $(d=0.68,95 \%$ CI $0.44-0.91$, $z=5.68, p \leqslant 0.001)$. When adolescent studies were analysed separately, the effect size was also medium $(d=0.51,95 \%$ CI $0.21-0.82, z=3.28, p=0.001)$. Child studies produced a medium pooled effect size $(d=0.70$, 95\% CI 0.46-0.92, $z=5.86, p \leqslant 0.001)$.

\section{Additional analysis}

There was evidence of moderate heterogeneity in both AN $\left(I^{2}=63.1 \%\right)$ and ASD $\left(I^{2}=37.0 \%\right)$ studies. To 


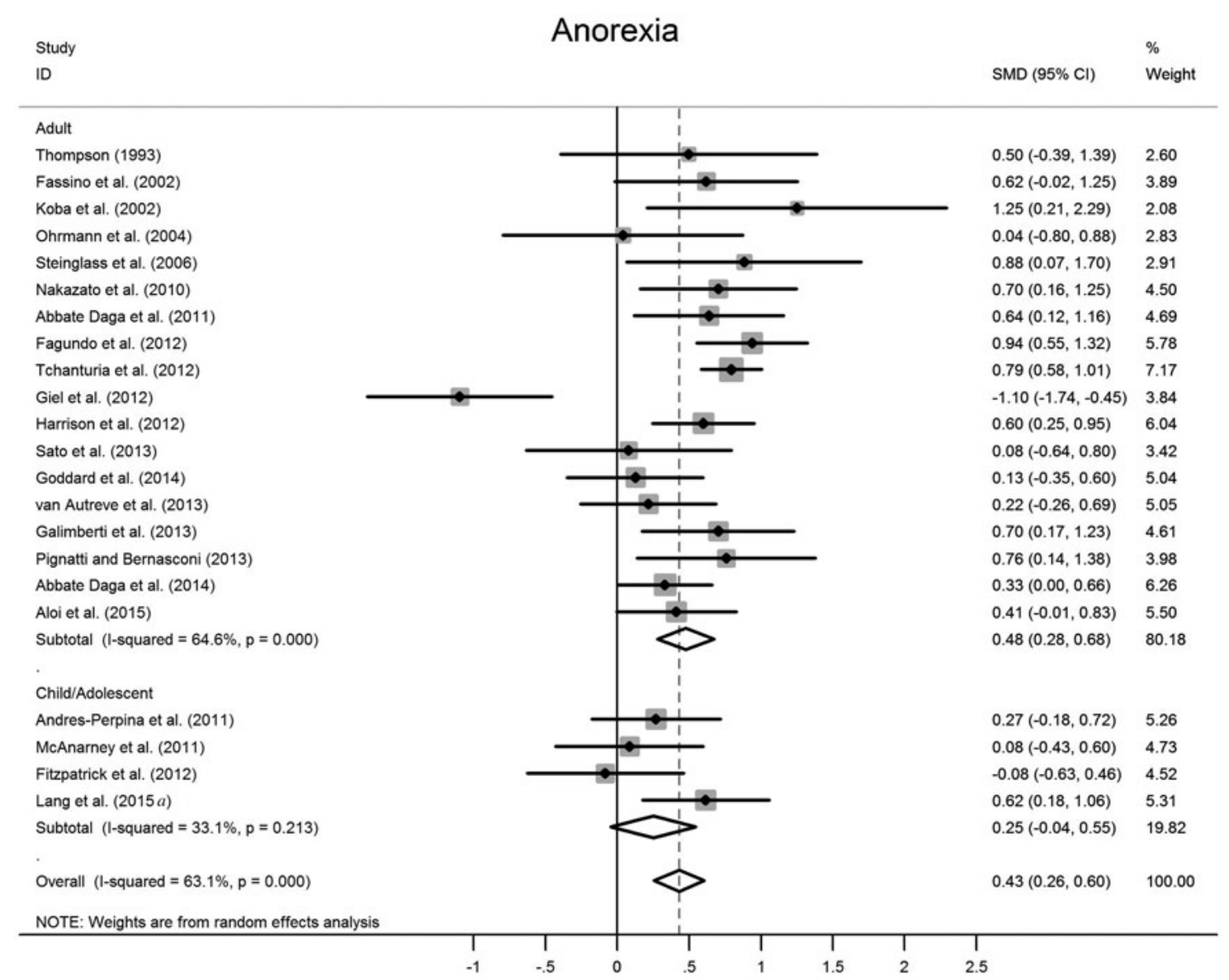

Fig. 2. Forest plot of mean perseverative error score on the Wisconsin Card Sorting Test: standardized mean effect size for differences (SMD) between anorexia nervosa and healthy controls. CI, Confidence interval.

examine the difference between ASD and AN groups, meta-regression was conducted with diagnosis as the moderator. Within adult studies, there was no effect of diagnosis and thus no significant difference between how ASD and AN groups performed on the WCST ( $n=28$, mean difference $=0.20,95 \%$ CI -0.17 to 0.56 , $t=1.12, p=0.274)$. There was an almost significant difference between the children and adolescent ASD and AN groups, with the ASD group scoring, on average, worse on the WCST than children and adolescents of the AN groups; however, this difference did not reach significance (mean difference $=0.44,95 \%$ CI -0.007 to $0.89, n=14, t=2.13, p=0.053$ ).

\section{Discussion}

The aim of this review was to synthesize the literature on the use of the WCST in both ASD and AN to examine whether there are differences between children and adults both within and between these clinical populations. There were a number of differences between studies including how a diagnosis of either AN or
ASD was given, the service and country in which participants were recruited from, the experimental paradigm used and how HCs were matched to the clinical groups. These differences may have made an impact on the individual outcomes of each study; however, generally the quality of the studies was deemed sufficient for meaningful synthesis and analyses. Studies also differed on the version of the WCST used, for example $128 v .64$ cards and computerized $v$. pen-paper versions. This may have had an impact on the results of each study. Children with ASD have been found to perform better on the computerized version of the test, suggesting that the versions are not equivalent and may measure slightly different constructs (Ozonoff, 1995).

The meta-analysis indicated significant differences between participants with AN and HCs, with the AN group making significantly more perseverative errors during the WCST. When children and adults data were analysed separately, the difference between children with AN and HCs was not significant, while in adults the difference remained significant, with a 


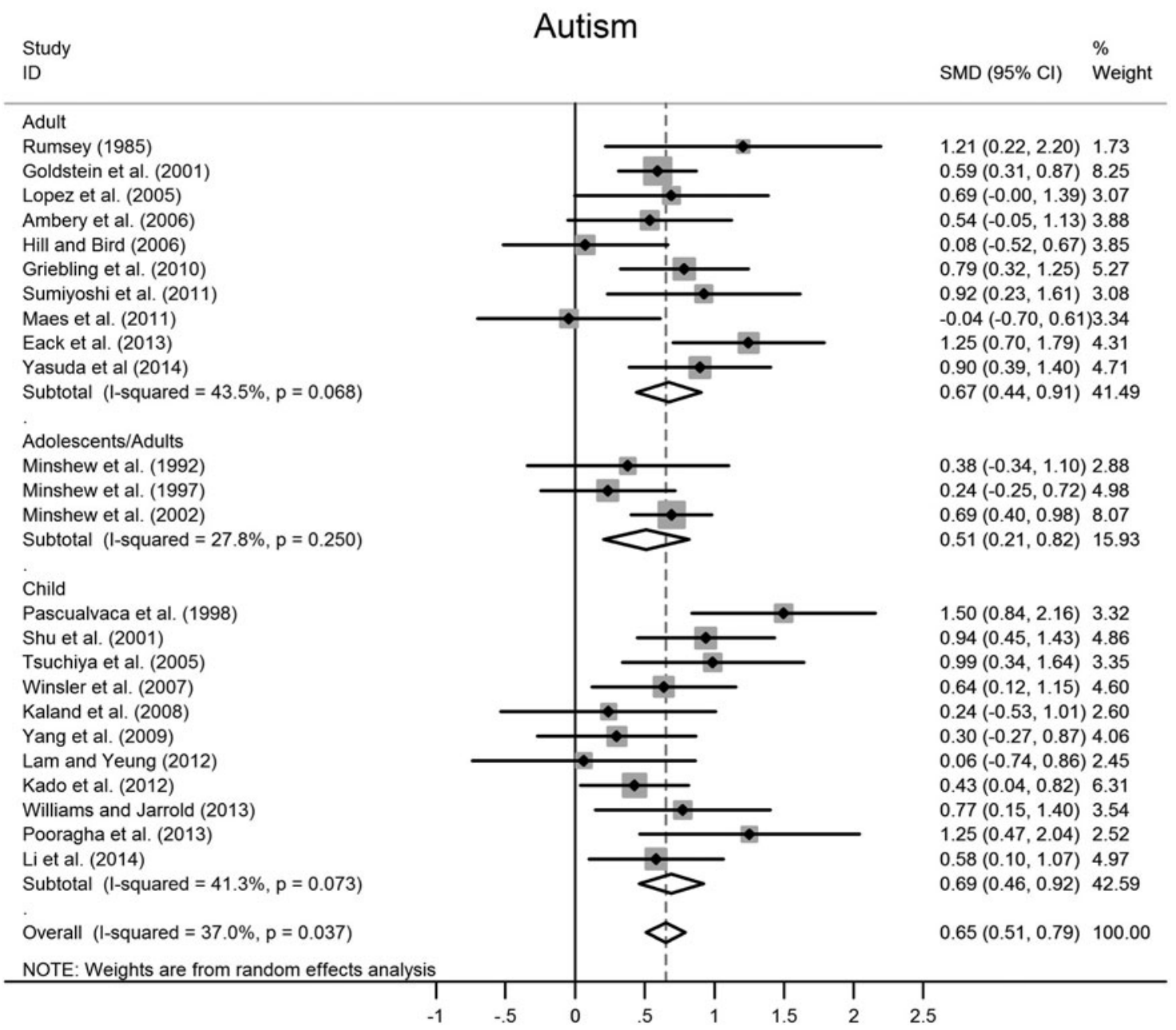

Fig. 3. Forest plot of mean perseverative error score on the Wisconsin Card Sorting Test: standardized mean effect size for differences (SMD) between autism spectrum disorder and healthy controls. CI, Confidence interval.

medium effect size. This indicates that adults with AN may have greater difficulty with set-shifting than young people with the disorder. This finding supports and extends a previous systematic review and meta-analysis (Roberts et al. 2007) which showed that adults with AN had significant difficulties with setshifting relative to HCs. The addition of thirteen studies since Roberts et al.'s (2007) meta-analysis has confirmed the original finding of a significant difference between adults with AN and HCs.

The addition of Lang et al.'s (2015a) empirical study to the child literature indicates a small non-significant effect of AN on set-shifting, in line with a previous systematic review (Lang et al. 2014). This non-significant difference between AN and HC groups may be driven by a lack of statistical power with a limited number of studies conducted with small numbers of participants. The Lang et al. (2015a) study was designed to specifically replicate the methods used within adult studies and to address previous methodological shortcomings in the child literature and therefore adds strength to the current literature, despite the result remaining nonsignificant. The analysis presented here shows that young people with AN may have difficulties with setshifting, but further studies are needed to determine whether these difficulties are significant. The effect size in adult studies is larger than that in child/adolescent studies and longitudinal studies are required to properly test whether there is a magnification of the effect over time, whereby poor set-shifting is a risk factor for AN, but is also intensified by the illness, thus acting as a precipitating and perpetuating factor within the disorder. Another possibility is that more severe setshifting problems during childhood are predictors of the persistence of these problems into adulthood. Alternatively, the WCST may be more reliable and sensitive to problems when used with adults, thus yielding bigger, significant effect sizes in adults.

Meta-analysis of ASD studies indicated a different pattern to the AN studies. Adults with ASD made 
significantly more perseverative errors on the WCST in comparison with HCs, indicating significant difficulties with set-shifting with a medium effect size. When child studies were analysed separately, the effect size was also medium, demonstrating that difficulties with set-shifting in ASD appear to be stable across the lifespan. This is a novel finding, as little attention has been paid in the ASD literature to WCST performance across the lifespan; however, these findings support previous work that suggests that deficits on the WCST are stable over time (Huizinga \& Van Der Molen, 2007) and studies of executive functioning deficits in children with ASD (Pellicano, 2007; Kimhi et al. 2014). The recent meta-analysis by Landry \& Al-Taie (2016) found age to be negatively correlated with set-shifting ability. While planned statistical analysis of our review did not specifically test this, CIs for the child and adult studies overlap so we are unable to conclude that there is a significant difference between children and adults with ASD.

Despite difficulties in set-shifting being widely reported within the ASD literature, preserved performance relative to HCs has also been documented in some studies (Ozonoff \& Jensen, 1999; Russell \& Hill, 2001). Hill (2004) attributes this discrepancy to methodological inconsistencies amongst studies; however, as the meta-analysis performed here only included studies using the WCST, it appears to provide strong evidence than individuals with ASD have problems in set-shifting, at least as assessed using the WCST. This is the first meta-analysis to statistically compare both adult and child ASD studies using the WCST and suggests very little difference between the two groups.

The meta-regression, analysing the impact of diagnosis on set-shifting ability, found no significant difference between ASD and AN, in either the adult or child/ adolescent groups, although there was a nonsignificant trend for children with ASD to perform worse than children with AN. This suggests that there may be differences in the development of setshifting difficulties across ages between $\mathrm{AN}$ and ASD, although larger, stronger-powered longitudinal studies would be needed to confirm this.

It is important to consider the implications of these meta-analyses for both AN and ASD populations. Within AN, interventions like cognitive remediation therapy (CRT) which targets cognitive processes have been developed and empirically tested (Tchanturia et al. 2013a, 2014, 2015), with improvements in setshifting as well as high acceptability and engagement in the treatment being reported. In spite of this success, the use of executive function training in ASD is limited, with a paucity of research in this area (De Vries et al. 2015). The few studies that have focused on flexibility training in ASD have yielded mixed results, with one study reporting improvements in flexibility (Kenworthy et al. 2014), one showing no improvement (Fisher \& Happe, 2005) and one suggesting a trend towards improvement with intention-to-treat analysis (De Vries et al. 2015). These mixed findings may suggest that the type of flexibility training currently offered to individuals with ASD is not suitable for this population. Trials in CRT for ASD are in their infancy but the success of CRT in other disorders along with the observation that children with ASD show development in executive functioning (Pellicano, 2010) makes it a worthwhile focus for future research.

While this review suggests that difficulties with setshifting are not directly comparable in ASD and AN, its impact on symptoms and wider functioning is still unknown. One of the main cognitive theories of ASD proposes that deficits in executive function underlie the disorder (Russell, 1997) and that these difficulties may also cause problems with social interaction and inflexible behaviour (Pellicano, 2007; Geurts et al. 2014). In AN, the cognitive-interpersonal maintenance model of AN (Treasure \& Schmidt, 2013) postulates that difficulty with flexibility is a pre-disposing factor for AN, making individuals more susceptible to societal rules and physical appearance. Thus, set-shifting may underlie other symptoms associated with the disorder. The observation that difficulties with set-shifting may underlie other symptoms in both ASD and AN suggests that transdiagnostic interventions which target cognitive style rather than direct symptoms could improve functioning and engagement in other therapies, such as cognitive-behavioural approaches which require a more flexible thinking style (Treasure et al. 2015).

This comparison between AN and ASD studies should, however, be interpreted in light of study limitations. The majority of ASD studies only included male participants whereas most AN studies included only females. Thus, there may be important gender differences within each disorder which make an impact on set-shifting ability. For example, in one study adolescent females with ASD outperformed their male counterparts on a test of set-shifting ability (Bolte et al. 2011); so if females with ASD were directly compared with females with AN, the results may be different. As starvation is thought to confound set-shifting ability in AN, it is also important to consider variables such as BMI and illness duration on WCST performance, which were not analysed in this review. A study in healthy female participants found set-shifting ability to be impaired after fasting for a $16 \mathrm{~h}$ period (Bolton et al. 2014), supporting the notion that in females at least, set-shifting is worsened following starvation. Thus, the question of whether poor set-shifting 
ability is a trait or state characteristic remains unanswered, despite the evidence from this review suggesting that this difficulty is present in young people with AN. In the future, population-based data, rather than data from clinical samples regarding set-shifting difficulties in those with either subclinical AN or ASD traits, may be useful to avoid bias. The impact of diagnosis was not investigated here and as the diagnostic criteria of ASD and AN have become broader under the revised DSM-V criteria (American Psychiatric Association, 2013), it is possible that greater heterogeneity between studies exists, making studies less comparable.

\section{Acknowledgements}

H.W. and K.T. would like to thank the Medical Research Council, Swiss Anorexia Foundation and the Psychiatry Research Trust for their support. D.S. received financial support from the National Institute of Health Research Biomedical Research Centre at South London and Maudsley NHS Foundation Trust and the Institute of Psychiatry, Psychology \& Neuroscience, King's College London.

This research received no specific grant from any funding agency, commercial or not-for-profit sectors.

\section{Declaration of Interest}

None.

\section{References}

Abbate-Daga G, Buzzichelli S, Amianto F, Rocca G, Marzola E, McClintock SM, Fassiono S (2011). Cognitive flexibility in verbal and nonverbal domains and decision making in anorexia nervosa patients: a pilot study. BMC Psychiatry

11, 162.

Abbate-Daga G, Buzzichelli S, Marzola E, Amianto F, Fassino S (2014). Clinical investigation of set-shifting subtypes in anorexia nervosa. Psychiatry Research 219, 592-597.

Allely C (2013). Anorexia nervosa - on the autistic spectrum? Psychologist 26, 656-658.

Aloi M, Rania M, Caroleo M, Bruni A, Palmieri A, Cauteruccio M, De Fazio P, Segura-Garcia C (2015). Decision making, central coherence and set-shifting: a comparison between binge eating disorder, anorexia nervosa and healthy controls. BMC Psychiatry 15, 6.

Ambery FZ, Russell AJ, Perry K, Morris R, Murphy DG (2006). Neuropsychological functioning in adults with Asperger syndrome. Autism 10, 551-564.

American Psychiatric Association (2013). Diagnostic and Statistical Manual of Mental Disorders, 5th edn. American Psychiatric Association: Washington, DC.

Andres-Perpina S, Lozano-Serra E, Puig O, Lera-Miguel S, Lazaro L, Castro-Fornieles J (2011). Clinical and biological correlates of adolescent anorexia nervosa with impaired cognitive profile. European Child and Adolescent Psychiatry 20, 541-549.

Atkinson TM, Ryan JP (2008). The use of variants of the trail making test in serial assessment: a construct validity study. Journal of Psychoeducational Assessment 26, 42-54.

Austin MP, Mitchell P, Wilhelm K, Parker G, Hickie I, Brodaty H, Chan J, Eyers K, Milic M, Hadzi-Pavlovic D (1999). Cognitive function in depression: a distinct pattern of frontal impairment in melancholia? Psychological Medicine 29, 73-85.

Baron-Cohen S, Jaffa T, Davies S, Auyeung B, Allison C, Wheelwright $S$ (2013). Do girls with anorexia nervosa have elevated autistic traits? Molecular Autism 4, 24.

Bolte S, Duketis E, Poustka F, Holtmann M (2011). Sex differences in cognitive domains and their clinical correlates in higher-functioning autism spectrum disorders. Autism 15, 497-511.

Bolton HM, Burgess PW, Gilbert SJ, Serpell L (2014). Increased set shifting costs in fasted healthy volunteers. PLOS ONE 9, e101946.

Borenstein M, Hedges LV, Higgins JPT, Rothstein HR (2009). Introduction to Meta-Analysis. Wiley: Chichester.

Bradburn MJ, Deeks JJ, Altman DG (1998). sbe24: metan an alternative meta-analysis command. Stata Technical Bulletin, 44, 4-15. Reprinted in Stata Technical Bulletin Reprints 8, 86-100. College Station, TX: Stata Press.

Cohen J (1988). Statistical Power Analysis for the Behavioral Sciences, 2nd edn. Laurence Erlbaum Associates: London.

Courty A, Maria AS, Lalanne C, Ringuenet D, Vindreau C, Chevallier C, Pouga L, Pinabel F, Philippe A, Adrien JL, Barry C, Berthoz S (2013). Levels of autistic traits in anorexia nervosa: a comparative psychometric study. BMC Psychiatry 13, 222.

Critical Appraisal Skills Programme (2013). CASP Case Control Checklist. Critical Appraisal Skills Programme. CASP: Oxford (http://media.wix.com/ugd/dded87_ 63fb65dd4e0548e2bfd0a982295f839e.pdf). Accessed March 2016.

Danner UN, Sanders N, Smeets PA, Van Meer F, Adan RA, Hoek HW, Van Elburg AA (2012). Neuropsychological weaknesses in anorexia nervosa: set-shifting, central coherence, and decision making in currently ill and recovered women. International Journal of Eating Disorders 45, 685-694.

De Vries M, Prins PJ, Schmand BA, Geurts HM (2015). Working memory and cognitive flexibility-training for children with an autism spectrum disorder: a randomized controlled trial. Journal of Child Psychology and Psychiatry 56, 566-576.

Eack SM, Bahorik AL, McKnight SA, Hogarty SS, Greenwald DP, Newhill CE, Phillips ML, Keshavan MS, Minshew NJ (2013). Commonalities in social and nonsocial cognitive impairments in adults with autism spectrum disorder and schizophrenia. Schizophrenia Research 148, 24-28.

Egger M, Davey Smith G, Schneider M, Minder C (1997). Bias in meta-analysis detected by a simple, graphical test. British Medical Journal 315, 629-634. 
Fagundo AB, De La Torre R, Jimenez-Murcia S, Aguera Z, Granero R, Tarrega S, Botella C, Banos R, Fernandez-Real JM, Rodriguez R, Forcano L, Fruhbeck G, GomezAmbrosi J, Tinahones FJ, Fernandez-Garcia JC, Casanueva FF, Fernandez-Aranda F (2012). Executive functions profile in extreme eating/weight conditions: from anorexia nervosa to obesity. PLOS ONE 7, e43382.

Fairburn CG, Cooper Z (1993). The eating disorder examination. In Binge Eating: Nature, Assessment and Treatment, 12th edn. (ed. C.G. Fairburn and G.T. Wilson), pp. 317-360. Guilford Press: New York.

Fassino S, Piero A, Daga GA, Leombruni P, Mortara P, Rovera GG (2002). Attentional biases and frontal functioning in anorexia nervosa. International Journal of Eating Disorders 31, 274-283.

Fisher N, Happe F (2005). A training study of theory of mind and executive function in children with autistic spectrum disorders. Journal of Autism and Developmental Disorders 35, 757-771.

Fitzpatrick KK, Darcy A, Colborn D, Gudorf C, Lock J (2012). Set-shifting among adolescents with anorexia nervosa. International Journal of Eating Disorders 45, 909-912.

Galimberti E, Fadda E, Cavallini MC, Martoni RM, Erzegovesi S, Bellodi L (2013). Executive functioning in anorexia nervosa patients and their unaffected relatives. Psychiatry Research 208, 238-244.

Geurts HM, Corbett B, Solomon M (2009). The paradox of cognitive flexibility in autism. Trends in Cognitive Science 13, 74-82.

Geurts HM, De Vries M, Van Den Beurgh SFWM (2014). Executive functioning theory of autism. In Handbook of Executive Functioning (ed. S. Goldstein and J.A. Nagleiri), pp. 121-141. Springer: New York.

Giel KE, Wittorf A, Wolkenstein L, Klingberg S, Drimmer E, Schoenenberg M, Rapp AM, Fallgatter AJ, Hautzinger M, Zipfel S (2012). Is impaired set-shifting a feature of "pure" anorexia nervosa? Investigating the role of depression in set-shifting ability in anorexia nervosa and unipolar depression. Psychiatry Research 200, 538-543.

Gillberg IC, Billstedt E, Wentz E, Anckarsater H, Rastam M, Gillberg C (2010). Attention, executive functions, and mentalizing in anorexia nervosa eighteen years after onset of eating disorder. Journal of Clinical Experimental Neuropsychology 32, 358-365.

Goddard E, Carral-Fernández L, Denneny E, Campbell IC, Treasure J (2014). Cognitive flexibility, central coherence and social emotional processing in males with an eating disorder. World Journal of Biological Psychiatry 15, 317-326.

Goldstein G, Johnson CR, Minshew NJ (2001). Attentional processes in autism. Journal of Autism and Developmental Disorders 31, 433-440.

Greve KW, Ingram F, Bianchini KJ (1998). Latent structure of the Wisconsin Card Sorting Test in a clinical sample. Archives of Clinical Neuropsychology 13, 597-609.

Greve KW, Stickle TR, Love JM, Bianchini KJ, Stanford MS (2005). Latent structure of the Wisconsin Card Sorting Test: a confirmatory factor analytic study. Archives of Clinical Neuropsychology 20, 355-364.
Griebling J, Minshew NJ, Bodner K, Libove R, Bansal R, Konasale P, Keshavan MS, Hardan A (2010). Dorsolateral prefrontal cortex magnetic resonance imaging measurements and cognitive performance in autism. Journal of Child Neurology 25, 856-863.

Harrison A, Tchanturia K, Naumann U, Treasure J (2012). Social emotional functioning and cognitive styles in eating disorders. British Journal of Clinical Psychology 51, 261-279.

Heaton RK, Chelune GJ, Talley JL, Kay GG, Curtiss G (1993). Wisconsin Card Sorting Test Manual. Psychological Assessment Resources, Inc.: Odessa, FL.

Higgins JP, Thompson SG, Deeks JJ, Altman DG (2003). Measuring inconsistency in meta-analyses. British Medical Journal 327, 557-560.

Hill EL (2004). Executive dysfunction in autism. Trends in Cognitive Science 8, 26-32.

Hill EL, Bird CM (2006). Executive processes in Asperger syndrome: patterns of performance in a multiple case series. Neuropsychologia 44, 2822-2835.

Holliday J, Tchanturia K, Landau S, Collier D, Treasure J (2005). Is impaired set-shifting an endophenotype of anorexia nervosa? American Journal of Psychiatry 162, 2269-2275.

Holmén A, Juuhl-Langseth M, Thormodsen R, Sundet K, Melle I, Rund BR (2012). Executive function tests in earlyonset psychosis: which one to choose? Scandinavian Journal of Psychology 53, 200-225.

Huizinga M, Van Der Molen MW (2007). Age-group differences in set-switching and set-maintenance on the Wisconsin Card Sorting Task. Developmental Neuropsychology 31, 193-215.

Huke V, Turk J, Saeidi S, Kent A, Morgan JF (2013). Autism spectrum disorders in eating disorder populations: a systematic review. European Eating Disorders Review 21, 345-351.

Huke V, Turk J, Saeidi S, Kent A, Morgan JF (2014). The clinical implications of high levels of autism spectrum disorder features in anorexia nervosa: a pilot study. European Eating Disorders Review 22, 116-121.

Kado Y, Sanada S, Yanagihara M, Ogino T, Ohno S, Watanabe K, Nakano K, Morooka T, Oka M, Ohtsuka Y (2012). Executive function in children with pervasive developmental disorder and attention-deficit/hyperactivity disorder assessed by the Keio version of the Wisconsin Card Sorting Test. Brain and Development 34, 354-359.

Kaland N, Smith L, Mortensen EL (2008). Brief report: cognitive flexibility and focused attention in children and adolescents with Asperger syndrome or high-functioning autism as measured on the computerized version of the Wisconsin Card Sorting Test. Journal of Autism and Developmental Disorders 38, 1161-1165.

Kenworthy L, Anthony LG, Naiman DQ, Cannon L, Wills MC, Luong-Tran C, Werner MA, Alexander KC, Strang J, Bal E, Sokoloff JL, Wallace GL (2014). Randomized controlled effectiveness trial of executive function intervention for children on the autism spectrum. Journal of Child Psychology and Psychiatry 55, 374-383.

Kenworthy L, Yerys BE, Anthony LG, Wallace GL (2008). Understanding executive control in autism spectrum 
disorders in the lab and in the real world. Neuropsychology Review 18, 320-338.

Kimhi Y, Shoam-Kugelmas D, Agam Ben-Artzi G, BenMoshe I, Bauminger-Zviely N (2014). Theory of mind and executive function in preschoolers with typical development versus intellectually able preschoolers with autism spectrum disorder. Journal of Autism and Developmental Disorders 44, 2341-2354.

Koba T, Shrie S, Nabeta Y (2002). Impaired performance of Wisconsin Card Sorting Test in patients with eating disorders: a preliminary study. Seishin Igaku 44, 681-683.

Lam YG, Yeung SSS (2012). Cognitive deficits and symbolic play in preschoolers with autism. Research in Autism Spectrum Disorders 6, 560-564.

Landry O, Al-Taie S (2016). A meta-analysis of the Wisconsin Card Sorting Task in autism. Journal of Autism and Developmental Disorders 46, 1220-1235.

Lang K, Lloyd S, Khondoker M, Simic M, Treasure J, Tchanturia K (2015a). Do children and adolescents with anorexia nervosa display an inefficient cognitive processing style? PLOS ONE 10, e0131724.

Lang K, Stahl D, Espie J, Treasure J, Tchanturia K (2014). Set shifting in children and adolescents with anorexia nervosa: an exploratory systematic review and meta-analysis. International Journal of Eating Disorders 47, 394-399.

Lang K, Treasure J, Tchanturia K (2015b). Is inefficient cognitive processing in anorexia a familial trait? A neuropsychological pilot study of mothers of offspring with a diagnosis of anorexia nervosa. World Journal of Biological Psychiatry. Published online 1 December 2015. doi:10.3109/ 15622975.2015.1112035.

Leopold R, Backenstrass M (2015). Neuropsychological differences between obsessive-compulsive washers and checkers: a systematic review and meta-analysis. Journal of Anxiety Disorders 30, 48-58.

Leung RC, Zakzanis KK (2014). Brief report: cognitive flexibility in autism spectrum disorders: a quantitative review. Journal of Autism and Developmental Disorders 44, 2628-2645.

Lezak MD (1995). Neuropsychological Assessment, 3rd edn. Oxford University Press: New York.

Li J, Zhu L, Liu J, Li X (2014). Social and non-social deficits in children with high-functioning autism and their cooperative behaviors. Research in Autism Spectrum Disorders 8, 1657-1671.

Lindner SE, Fichter MM, Quadflieg N (2014). Set-shifting and its relation to clinical and personality variables in full recovery of anorexia nervosa. European Eating Disorders Review 22, 252-259.

Lopez BR, Lincoln AJ, Ozonoff S, Lai Z (2005). Examining the relationship between executive functions and restricted, repetitive symptoms of autistic disorder. Journal of Autism and Developmental Disorders 35, 445-460.

Maes JHR, Eling PATM, Wezenberg E, Vissers CTWM, Kan CC (2011). Attentional set shifting in autism spectrum disorder: differentiating between the role of perseveration, learned irrelevance, and novelty processing. Journal of Clinical and Experimental Neuropsychology 33, 210-217.
McAnarney ER, Zarcone J, Singh P, Michels J, Welsh S, Litteer T, Wang H, Klein JD (2011). Restrictive anorexia nervosa and set-shifting in adolescents: a biobehavioral interface. Journal of Adolescent Health 49, 99-101.

Milner B (1963). Effects of different brain lesions on card sorting. Archives of Neurology 9, 90-100.

Minshew NJ, Goldstein G, Siegel DJ (1997). Neuropsychologic functioning in autism: profile of a complex information processing disorder. Journal of International Neuropsychological Society 3, 303-316.

Minshew NJ, Meyer J, Goldstein G (2002). Abstract reasoning in autism: a dissociation between concept formation and concept identification. Neuropsychology 16, 327-334.

Minshew NJ, Muenz LR, Goldstein G, Payton JB (1992). Neuropsychological functioning in nonmentally-retarded autistic individuals. Journal of Clinical and Experimental Neuropsychology 14, 749-761.

Moher D, Liberati A, Tetzlaff J, Altman DG, Group P (2009). Preferred Reporting Items for Systematic Reviews and Meta-Analyses: the PRISMA statement. British Medical Journal 339, b2535.

Nakazato M, Hashimoto K, Schmidt U, Tchanturia K, Campbell IC, Collier DA, Iyo M, Treasure J (2010). Serum glutamine, set-shifting ability and anorexia nervosa. Annals of General Psychiatry 9, 29.

Nelson HE (1976). A modified card sorting test sensitive to frontal lobe defects. Cortex 12, 313-314.

Nielsen S, Anckarsätar H, Gillberg C, Rastam M, Wentz E (2015). Effects of autism spectrum disorders on outcome in teenage-onset anorexia-nervosa evaluated by the MorganRussell Outcome Assessment Schedule: a controlled community-based sample. Molecular Autism 6, 14.

Odent M (2010). Autism and anorexia nervosa: two facets of the same disease? Medical Hypotheses 75, 79-81.

Ohrmann P, Kersting A, Suslow T, Lalee-Mentzel J, Donges US, Fiebich M, Arolt V, Heindel W, Pfleiderer B (2004). Proton magnetic resonance spectroscopy in anorexia nervosa: correlations with cognition. Neuroreport 15, 549-553.

Oldershaw A, Hambrook D, Tchanturia K, Treasure J, Schmidt U (2010). Emotional theory of mind and emotional awareness in recovered anorexia nervosa patients. Psychosomatic Medicine 72, 73-79.

Oldershaw A, Treasure J, Hambrook D, Tchanturia K, Schmidt U (2011). Is anorexia nervosa a version of autism spectrum disorders? European Eating Disorders Review 19, 462-474.

Ozonoff S (1995). Reliability and validity of the Wisconsin Card Sorting Test in studies of autism. Neuropsychology $\mathbf{9}$, 491-500.

Ozonoff S, Jensen J (1999). Brief report: specific executive function profiles in three neurodevelopmental disorders. Journal of Autism and Developmental Disorders 29, 171-177.

Pantelis C, Barber FZ, Barnes TR, Nelson HE, Owen AM, Robbins TW (1999). Comparison of set-shifting ability in patients with chronic schizophrenia and frontal lobe damage. Schizophrenia Research 37, 251-270.

Pascualvaca DM, Fantie BD, Papageorgiou M, Mirsky AF (1998). Attentional capacities in children with autism: is 
there a general deficit in shifting focus? Journal of Autism and Developmental Disorders 28, 467-478.

Pellicano E (2007). Links between theory of mind and executive function in young children with autism: clues to developmental primacy. Developmental Psychology 43, 974-990.

Pellicano E (2010). The development of core cognitive skills in autism: a 3-year prospective study. Child Development 81, 1400-1416.

Pellicano L, Hiller R (2013). Anorexia and autism: a cautionary note. Psychologist 26, 780.

Pender S, Gilbert SJ, Serpell L (2014). The neuropsychology of starvation: set-shifting and central coherence in a fasted nonclinical sample. PLOS ONE 9, e110743.

Pignatti R, Bernasconi V (2013). Personality, clinical features, and test instructions can affect executive functions in eating disorders. Eating Behaviour 14, 233-236.

Pooragha F, Kafi SM, Sotodeh SO (2013). Comparing response inhibition and flexibility for two components of executive functioning in children with autism spectrum disorder and normal children. Iranian Journal of Pediatrics 23, 309-314.

Rhind C, Bonfioli E, Hibbs R, Goddard E, Macdonald P, Gowers S, Schmidt U, Tchanturia K, Micali N, Treasure J (2014). An examination of autism spectrum traits in adolescents with anorexia nervosa and their parents. Molecular Autism 5, 56.

Roberts ME, Tchanturia K, Stahl D, Southgate L, Treasure J (2007). A systematic review and meta-analysis of setshifting ability in eating disorders. Psychological Medicine 37, 1075-1084.

Roberts ME, Tchanturia K, Treasure JL (2010). Exploring the neurocognitive signature of poor set-shifting in anorexia and bulimia nervosa. Journal of Psychiatric Research 44, 964-970.

Rumsey JM (1985). Conceptual problem-solving in highly verbal, nonretarded autistic men. Journal of Autism and Developmental Disorders 15, 23-36.

Russell J (1997). Autism as an Executive Disorder. Oxford University Press: New York.

Russell J, Hill EL (2001). Action-monitoring and intention reporting in children with autism. Journal of Child Psychology and Psychiatry and Allied Disciplines 42, 317-328.

Sanders J, Johnson KA, Garavan H, Gill M, Gallagher L (2008). A review of neuropsychological and neuroimaging research in autistic spectrum disorders: attention, inhibition and cognitive flexibility. Research in Autism Spectrum Disorders 2, 1-16.

Sato Y, Saito N, Utsumi A, Aizawa E, Shoji T, Izumiyama M, Mushiake H, Hongo M, Fukudo S (2013). Neural basis of impaired cognitive flexibility in patients with anorexia nervosa. PLOS ONE 8, e61108.

Sharp S (1998). sbe23: meta-analysis regression. Stata Technical Bulletin, 42, 16-22. Reprinted in Stata Technical Bulletin Reprints 7, 148-155. College Station, TX: Stata Press.

Shu BC, Lung FW, Tien AY, Chen BC (2001). Executive function deficits in non-retarded autistic children. Autism 5, 165-174.
Steichen TJ (1998). sbe19: tests for publication bias in metaanalysis. Stata Technical Bulletin, 41, 9-15. Reprinted in Stata Technical Bulletin Reprints 7, 125-133. College Station, TX: Stata Press.

Steinglass JE, Walsh BT, Stern Y (2006). Set shifting deficit in anorexia nervosa. Journal of International Neuropsychological Society 12, 431-435.

Sumiyoshi C, Kawakubo Y, Suga M, Sumiyoshi T, Kasai K (2011). Impaired ability to organize information in individuals with autism spectrum disorders and their siblings. Neuroscience Research 69, 252-257.

Tchanturia K (editor) (2015). Cognitive Remediation Therapy (CRT) for Eating and Weight Disorders. Routledge: Hove, East Sussex.

Tchanturia K, Davies H, Roberts M, Harrison A, Nakazato M, Schmidt U, Treasure J, Morris R (2012). Poor cognitive flexibility in eating disorders: examining the evidence using the Wisconsin Card Sorting Task. PLOS ONE 7, e28331.

Tchanturia K, Lloyd S, Lang K (2013a). Cognitive remediation therapy for anorexia nervosa: current evidence and future directions. International Journal of Eating Disorders 46, 492-495.

Tchanturia K, Lounes N, Holttum S (2014). Cognitive remediation in anorexia nervosa and related conditions: a systematic review. European Eating Disorders Review 22, 454-462.

Tchanturia K, Morris RG, Anderluh MB, Collier DA, Nikolaou V, Treasure J (2004). Set shifting in anorexia nervosa: an examination before and after weight gain, in full recovery and relationship to childhood and adult OCPD traits. Journal of Psychiatric Research 38, 545-552.

Tchanturia K, Smith E, Weineck F, Fidanboylu E, Kern N, Treasure J, Baron Cohen S (2013b). Exploring autistic traits in anorexia: a clinical study. Molecular Autism 4, 44.

Thompson SBN (1993). Implications of neuropsychological test results of women in a new phase of anorexia nervosa. European Eating Disorders Review 1, 152-165.

Treasure J (2013). Coherence and other autistic spectrum traits and eating disorders: building from mechanism to treatment. The Birgit Olsson lecture. Nordic Journal of Psychiatry 67, 38-42.

Treasure J, Cardi V, Lappanen J, Turton R (2015). New treatment approaches for severe and enduring eating disorders. Physiology and Behavior 152, 456-465.

Treasure J, Schmidt U (2013). The cognitive-interpersonal maintenance model of anorexia nervosa revisited: a summary of the evidence for cognitive, socio-emotional and interpersonal predisposing and perpetuating factors. Journal of Eating Disorders 1, 13.

Tsuchiya E, Oki J, Yahara N, Fujieda K (2005). Computerized version of the Wisconsin Card Sorting Test in children with high-functioning autistic disorder or attention-deficit/ hyperactivity disorder. Brain and Development 27, 233-236.

Van Autreve S, De Baene W, Baeken C, Van Heeringen C, Vervaet M (2013). Do restrictive and bingeing/purging subtypes of anorexia nervosa differ on central coherence and set shifting? European Eating Disorders Review 21, 308-314. 
Westwood H, Eisler I, Mandy W, Leppanen J, Treasure J, Tchanturia K (2016). Using the autism-spectrum quotient to measure autistic traits in anorexia nervosa: a systematic review and meta-analysis. Journal of Autism and Developmental Disorders 46, 964-977.

Williams D, Jarrold C (2013). Assessing planning and setshifting abilities in autism: are experimenter-administered and computerised versions of tasks equivalent? Autism Research 6, 461-467.

Winsler A, Abar B, Feder MA, Schunn CD, Rubio DA (2007). Private speech and executive functioning among high-functioning children with autistic spectrum disorders. Journal of Autism and Developmental Disorders 37, 1617-1635.

Wu M, Brockmeyer T, Hartmann M, Skunde M, Herzog W, Friederich HC (2014). Set-shifting ability across the spectrum of eating disorders and in overweight and obesity: a systematic review and meta-analysis.

Psychological Medicine 44, 3365-3385.

Yang J, Zhou S, Yao S, Linyan S, McWhinnie C (2009). The relationship between theory of mind and executive function in a sample of children from mainland China. Child Psychiatry and Human Development 40, 169-182.

Yasuda Y, Hashimoto R, Ohi K, Yamamori H, Fujimoto M, Umeda-Yano S, Fujino H, Takeda M (2014). Cognitive inflexibility in Japanese adolescents and adults with autism spectrum disorders. World Journal of Psychiatry 4, 42-48.

Zucker N, Losh M, Bulik CM, LaBar KS, Piven J, Pelphrey KA (2007). Anorexia nervosa and autism spectrum disorders: guided investigation of social cognitive phenotypes. Psychological Bulletin 133, 976-1006. 